$$
N>1-20940
$$

NASA TECHNICAL MEMORANDUM

NASA TM X-52992

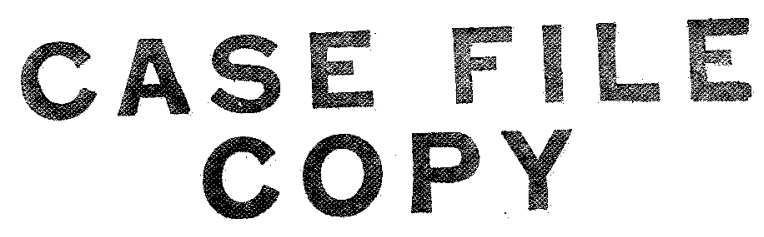

\title{
MATHEMATICAL REPRESENTATION OF CURRENT DENSITY PROFILES FROM ION THRUSTERS
}

by Thaine W. Reynolds

Lewis Research Center

Cleveland, Ohio

TECHNICAL PAPER proposed for presentation at Seventh Propulsion Joint Specialist Conference sponsored by the American Institute of Aeronautics and Astronautics Salt Lake City, Utah, June 14-18, 1971 


\author{
by Thaine W. Reynolds \\ Lewis Research Center \\ National Aeronautics and Space Administration \\ Cleveland, Ohio
}

\section{Summary}

A concise and convenient way of expressing ion beam spreading characteristics is presented. The twoparameter equation, $\left.j(z, \theta) /[j(z, 0)]=e^{-[\lambda(1-\cos \theta)]}\right]^{n}$, is shown to be capable of representing experimental beam current density profiles from several bombardment ion thrusters. This is a far-field expression. A calculation of the development of the profiles downstream of an extended source indicates that ion beam profiles should be taken at least eight thruster radii away to be representative of the far-field behavior of the beam.

Parametric plots (involving various combinations of $n$ and $\lambda$ ) of thrust factor, ion beam envelopes, and back-sputtering due to ion beam erosion illustrate the use of the ion beam equation in beam-interaction calculations. Experimental ion beams illustrated had thrust factors varying from 0.95 to near 1.0 .

\section{Introduction}

A description of the ion beam density profile of electron bombardment thrusters is needed for both mission analysis and spacecraft design. The angular spread of the ion beam as it leaves the thruster affects placement of possible spacecraft components, such as solar cells and thermal control surfaces, to avoid the problems of the ion beam striking sensitive surfaces and causing sputtering erosion. A loss in thrust is also involved when a significant fraction of the beam ions have trajectories away from the axis.

This report presents a two-parameter equation which has been found useful in expressing analytically the ion beam profile of a number of bombardment ion thrusters for which experimental profile data were available. The procedure for determining the values of the parameters for fitting the equation to experimental data is described. Finally, several calculations involving interactions of the beam profile are presented to illustrate its utility.

\section{Density Profile Equation}

Form of Equation

Ion beam density profiles from electron bombardment thrusters have been described as having a relatively flat central core and an exponential dropoff with increasing distance from the beam centerline. (1) An equation which exhibits the se characteristics is

$$
\frac{j(z, \theta)}{j(z, 0)}=e^{-[\lambda(1-\cos \theta)]^{n}}
$$

$j(z, \theta)$ current density in radial plane at axial distance $\mathrm{z}$ and radial angle $\theta$ (The current density is taken in the plane normal to the $z$-direction (radial plane) though the particle trajectories are at angles to this plane.)

$j(z, 0)$ current density in radial plane at axial distance $\mathrm{z}$ on centerline

$\theta$ half-angle from centerline to point of measurement

$\mathrm{n}, \lambda \quad$ parameters to be determined

(All symbols are defined in appendix A.)

This equation is deduced, then, simply from observation that experimentally determined profiles can be reasonably well described with the appropriate choice of the parameters $\mathrm{n}$ and $\lambda$. Equation (1) is analogous to a similarly derived expression for density profiles of supersonic jets expanding into a vacuum. (2)

Figure 1 shows the typical shapes of profiles obtained for $n=1$ and 2 and for several values of $\lambda$. A higher $\lambda$ for any given $\mathrm{n}$ indicates a more collimated beam. Similarly, a higher $\mathrm{n}$ for any given $\lambda$ indicates a more collimated beam.

Equation (1) is a far-field expression. It describes the expanding flow as if the ion trajectories were straight lines which appear to originate from a point source. The current density thus would vary along any trajectory inversely as the square of the distance from the source. The flow is completely defined as a function of the angle $\theta$. Equation (1) must necessarily become inapplicable close to the thruster. The distance from the thruster within which the equation is not useful is discussed in appendix $B$.

The total current at any axial location $\mathrm{z}$ must be constant and equal to the emitted current. Thus,

$$
\int_{0}^{\infty} 2 \pi r j(z, \theta) d r=\bar{j} \pi R_{0}^{2}
$$

Substitution of Eq. (1) into Eq. (2) and rearranging yield

$$
\frac{j(\mathrm{z}, 0)}{\overline{\mathrm{j}}}=\frac{\mathrm{R}_{0}^{2}}{2 \int_{0}^{\infty} \mathrm{e}^{-[\lambda(1-\cos \theta)]^{n}} \mathrm{rdr}}
$$

Letting

$$
\mathrm{y}=\frac{\mathrm{r}}{\mathrm{z}}
$$

where as shown in sketch (a), 
$\frac{j(z, 0)}{\bar{j}}=\left(\frac{1}{2 \int_{0}^{\infty} \exp \left\{-\left[\lambda\left(1-\frac{1}{\sqrt{1+y^{2}}}\right)\right]^{n}\right\} y d y}\right)\left(\frac{R_{0}}{z}\right)^{2}$

Designating the bracketed term in Eq. (5) as $c(n, \lambda)$,

$$
c(n, \lambda)=\frac{1}{2 \int_{0}^{\infty} \exp \left\{\left[\lambda\left(1-\frac{1}{\sqrt{1+y^{2}}}\right)\right]^{n}\right\} y d y}
$$

Eq. (5) can be expressed

$$
\frac{j(z, 0)}{\bar{j}}=c(n, \lambda) \frac{R_{0}^{2}}{z^{2}}
$$

Finally, Eqs. (7) and (1) combine to give the complete current density relation at any location,

$$
\frac{j(\mathrm{z}, \theta)}{\bar{j}}=\underbrace{c(n, \lambda) \frac{R_{0}^{2}}{z^{2}}}_{I} \underbrace{e^{-[\lambda(1-\cos \theta)]^{n}}}_{\text {II }}
$$

The part I of Eq. (8) gives the variation along the centerline; part II gives the radial variation. Values. of $\mathrm{c}(\mathrm{n}, \lambda)$ are plotted in Fig. 2.

\section{Comparison of Equation with Experimental Data}

The applicability of Eq. (1) to describing experimental ion beam profile data is shown in Figs. 3(a) to (g). In these figures the experimental profile data cited $(1,3-6)$ are shown by the data symbols. The solid lines are the plots of Eq. (1) for the particular values of $n$ and $\lambda$ cited on each curve. The value of $z / R_{0}$, the ratio of the distance downstream that the experimental profile was taken to the thruster radius, is also shown.

Two sets of experimental data for a given thruster have ion beam density profiles at two or more axial distances (Figs. 3(d) and (e)). In Fig. 3(d), the data at axial distance ratios $z / R_{0}$ of 14.8 and 25.2 fall on the same curve, showing that the assumption of linear trajectories between the two distances was applicable to this case. In Ref. 1, from which the data of Fig. 3(e) were obtained, the thruster size is not given. The actual linear distance at which the several profiles were taken is therefore shown in Fig. 3(e), rather than the distance ratios $\mathrm{z} / \mathbf{R}_{0}$ which are cited in the other plots. The profiles at axial distances of $16.5,21.5,28.4$, and 41.4 centimeters are closely approximated by the single profile equation with $\mathrm{n}=1.19$ and $\lambda=85$. The data of the closest profile $(\mathrm{z}=11.5 \mathrm{~cm})$ may be too close to the thruster to expect far-field behavior (see appendix B). The furthest downstream profile (at $\mathrm{z}=53.4 \mathrm{~cm}$ ) shows considerable asymmetry and is plotted separately in Fig. 3(e) along with the same equation plot as in the other part of this figure. The profiles of Fig. 3(e) also support the assumption of linear ion trajectories in the far field. Reasons for the asymmetry of the one profile were not discussed in Ref. 1 .
The other profiles of Fig. 3 were taken at distance ratios $\mathrm{z} / \mathrm{R}_{0}$ varying from 4 to 23.3 .

It can be seen that Eq. (1) is capable of representing the experimental, ion beam, current density profile data reasonably well. The greatest differences between experiment and equation values occur at the low-density values where the accuracy of determination is least.

Some of the information from Fig. 3 is summarized in table $I$.

\section{Determination of Parameter Values}

An experimental profile can be readily checked against Eq. (1) to determine the appropriate values of $\mathrm{n}$ and $\lambda$. The value of $\lambda$ is determined, independently of $n$, from the angle $\theta_{e}$ at which $j\left(z, \theta_{e}\right) /[j(z, 0)]=e^{-1}$ (i.e., 0.368),

$$
\lambda=\frac{1}{1-\cos \theta_{e}}
$$

Then, from any other experimental point, the value of $n$ is determined. For instance, at the angle $\theta_{0.1}$, at which $j\left(z, \theta_{0.1}\right) /(j(z, 0))=0.1$,

$$
\mathrm{n}=\frac{0.834}{\ln \left[\lambda\left(1-\cos \theta_{0.1}\right)\right]}
$$

The radial profile will thus fit at the three values of angle $\theta, 0, \theta_{e}$, and $\theta_{0.1}$.

\section{Application to Certain Problems}

\section{Thrust Factor}

The ratio of the axial component of thrust to the thrust that would be obtained if all trajectories were directed along the axis is referred to herein as the thrust factor $\mathbf{F}_{\mathbf{T}}$. Thus,

$$
\mathrm{F}_{\mathrm{T}}=\frac{\int_{0}^{\infty} \dot{\mathrm{m}}(\mathrm{r}) \mathrm{v} \cos \theta 2 \pi \mathrm{r} d \mathrm{r}}{\int_{0}^{\infty} \mathrm{m}(\mathrm{r}) \mathrm{v} 2 \pi \mathrm{r} \mathrm{dr}}
$$

The velocity $\mathrm{v}$ is assumed to be constant for any trajectory and to be directed in the $\theta$-direction. Equation (11) can be expressed, then, as

$$
\mathbf{F}_{T}=\frac{\int_{0}^{\infty} \frac{j(z, r)}{\bar{j}} \cos \theta r d r}{\int_{0}^{\infty} \frac{j(z, r)}{\bar{j}} r d r}
$$

Substituting Eqs. (1) and (4) into Eq. (12) yields

$$
\mathrm{F}_{\mathrm{T}}=\frac{\int_{0}^{\infty} \exp \left\{-\left[\lambda\left(1-\frac{1}{\sqrt{1+\mathrm{y}^{2}}}\right)\right]^{\mathrm{n}}\right\} \frac{\mathrm{y}}{\sqrt{1+\mathrm{y}^{2}}} d y}{\int_{0}^{\infty} \exp \left\{-\left(\lambda\left(1-\frac{1}{\sqrt{1+\mathrm{y}^{2}}}\right)\right]^{\mathrm{n}}\right\} \mathrm{ydy}}
$$


Numerical integration of Eq. (13) resulted in the values of thrust factor shown plotted in Fig. 4 .

\section{Ion Beam Envelope}

The fraction of the total ion beam enclosed within a given half-angle ${ }^{\theta} \mathrm{L}$ is expressed as

$\frac{I\left(\theta_{L}\right)}{I \text { (total })}=\frac{\int_{0}^{r=2 \tan \theta} L e^{-[\lambda(1-\cos \theta)]^{n} 2 \pi r d r}}{\int_{0}^{\infty} e^{-[\lambda(1-\cos \theta)]^{n} 2 \pi r d r}}$

Substituting Eqs. (4) and (6) into Eq. (14) yields $\frac{\mathrm{I}\left(\theta_{L}\right)}{\mathrm{I} \text { (total) }}=2 c(\mathrm{n}, \lambda) \int_{0}^{\mathrm{y}_{\mathrm{L}}} \exp \left\{-\left[\lambda\left(1-\frac{1}{\sqrt{1+\mathrm{y}^{2}}}\right)\right]^{\mathrm{n}}\right\} \mathrm{y} d \mathrm{y}$

Numerical integration of Eq. (15) for values of $n=1$ and 2 and for several $\lambda$-values yielded the curves shown in Figs. 5(a) and (b), for the fraction of the total beam contained within the angle $\theta^{\theta}$.

\section{Return of Sputtered Target Flux to Thruster}

The testing of ion thrusters in vacuum tanks causes sputtering of the facility walls where the ion beam impinges. The return of this sputtered material to the thruster face and condensation thereon was shown to affect the operation of glass-coated accelerator grids. (7) In order to get some estimate of the amount of backsputtered material to expect, the following model was used (sketch (b)), which made use of the proposed beam profile Eq. (1).

The returning (back sputtered) flux rate $\mu_{2}$ depends on the local emission rate of sputtered material $\nu_{1}$ and the view factor $\mathrm{dF}_{1,2}$ from the point of sputtering to the thruster center. (The variation across the thruster face will not be more than a few percent as long as the sputtered surface is about five or more thruster radii away.)

$$
\mu_{2}=\int_{\text {Area }} \nu_{1} \mathrm{dF}_{1,2}
$$

Assuming cosine-law distribution of the sputtered material and cylindrical symmetry, the back flux from the target and the wall can be written separately and summed. (View factors defined in appendix A are derivable from relations in Ref. 8.)

$\mu_{2}=2 z_{T}^{2} \int_{0}^{\mathrm{R}} \frac{\nu_{1}(\mathrm{r}) \mathrm{r} d \mathrm{r}}{\left(\mathrm{z}_{\mathrm{T}}^{2}+\mathrm{r}^{2}\right)^{2}}+2 \mathrm{R}^{2} \int_{0}^{\mathrm{z}_{\mathrm{T}} \mathrm{T}_{\nu_{1}(\mathrm{z}) \mathrm{z} \mathrm{dz}}} \frac{\left(\mathrm{R}^{2}+\mathrm{z}^{2}\right)^{2}}{\left(\int^{2}\right.}$

The sputtered emission rate $\nu_{1}$ depends on the incident ion rate $\mu_{1}$ and the sputtering ratio $\mathrm{S}$,

$$
\nu_{1}=\mu_{1} \mathrm{~s}
$$

In turn, $\mathrm{s}$ depends on incident ion energy, target material, and angle of incidence. The angular variation was assumed to follow the relation $(9)$

$$
\mathrm{S}=\mathbf{S}(0) \sec \alpha
$$

where $\alpha$ is the angle between the surface normal and the incident ion direction.

For the variation of $\mu_{1}$ with the position variables $z$ and $r$, use was made of Eq. (8).

It can be seen in sketch (a) that for the circular target $\alpha=\theta$, and that for the cylindrical tank wall $\alpha=(\pi / 2)-\theta$. Also the arrival rate on the cylindrical wall is related to the arrival rate on the target at the same location by

$$
\left[\mu_{1}(\mathrm{z}, \mathrm{R})\right]_{\text {Tank wall }}=\left[\mu_{1}(\mathrm{z}, \mathrm{R})\right]_{\text {Target }} \tan \theta
$$

By converting to the dimensionless variable $\mathrm{x}$, where

$$
\mathrm{x}=\frac{\mathrm{z}}{\mathrm{R}}
$$

and using relations (4), (8), and (18) to (21), Eq. (17) can be transformed into

$$
\begin{aligned}
& \frac{\mu_{2}}{\bar{\nu}}=2 \mathrm{~S}(0) \frac{\mathrm{R}_{0}^{2}}{\mathrm{z}_{\mathrm{T}}^{2}} \underbrace{\rho_{0}^{\mathrm{R} / \mathrm{z}} \mathrm{T}} \underbrace{\exp \left\{-\left[\lambda\left(1-\frac{1}{\sqrt{1+\mathrm{y}^{2}}}\right)\right]^{\mathrm{n}}\right\} \mathrm{y} d \mathrm{y}}_{\mathrm{I}} \\
& +2 \mathrm{~S}(0) \frac{\mathrm{R}_{0}^{2}}{\mathrm{R}^{2}} \underbrace{\mathrm{z} / \mathrm{R}}_{\mathrm{II}} \underbrace{\left.\exp \left\{-\left[\lambda\left(1-\frac{\mathrm{x}}{\sqrt{1+\mathrm{x}^{2}}}\right)\right]\right\}^{\mathrm{n}}\right\} \mathrm{dx}}
\end{aligned}
$$

Term I of Eq. (22) is the back flux from the circular target. Term II is the back flux from the cylindrical wall. The two parts of Eq. (22) are plotted separately in Figs. 6 and 7 for values of $\mathrm{n}=1$ and 2 and for several values of $\lambda$.

In Figs. 6(a) and (b), for example, the ordinate parameter

$$
\frac{\mu_{2}}{\bar{\nu}}\left(\frac{\mathrm{z}_{\mathrm{T}}}{\mathrm{R}_{0}}\right)^{2} \frac{1}{2 \mathrm{~S}(0)}
$$

is plotted. This parameter accounts for the flux ratio $\mu_{2} / \nu$, from a circular target of radius $r$ to the thruster exit center.

The return flux from a target is obtained, then, as

$$
\left(\frac{\mu_{2}}{\bar{\nu}}\right)_{\text {target }}=\left\{\frac{\mu_{2}}{\bar{\nu}} \frac{\mathrm{z}_{\mathrm{T}}^{2}}{\mathrm{R}_{0}^{2}} \frac{1}{2[\mathrm{~S}(0)]}\right\}_{\text {target }} \frac{\mathrm{R}_{0}^{2}}{\mathrm{z}} 2 \mathrm{~S}(0)
$$

It can be seen, for instance, that for $n=1$ and $\lambda=50$, no significant fraction of the total return flux from a circular target is obtained beyond about a $20^{\circ}$ half-angle. For $n=1$ and $\lambda=15$, most of the return 
flux is contined to the target that is within a 0 -value of about $+5^{\circ}$. For $n \div 2$ and these same $\lambda$-values, the cutoff angles are nearor $15^{\circ}$ and $30^{\circ}$, respeetivuly.

Figures 7(a) and (b) show the roturn flux from the tank walls to the thruster exit center. Here the ordinate gives the return flux from $x=0$ out to any axial distance $\mathrm{x}=\mathrm{z} / \mathrm{R}$. (The reforence radius $\mathrm{R}$ in these plots is the tank radius, not the thruster radius.) 'The return flux from the eylindrieal wall is then

$$
\left(\frac{\mu_{2}}{\bar{\nu}}\right)_{\text {wall }}=\left[\frac{\mu_{2}}{\bar{\nu}} \frac{\mathrm{R}^{2}}{\mathrm{R}_{0}^{2}} \frac{1}{2 \mathrm{~S}(0)}\right]_{\text {wall }} \frac{\mathrm{R}_{0}^{2}}{\mathrm{R}^{2}} 2 \mathrm{~S}(0)
$$

In this cuse it can be seen that for $n=1$ and $\lambda=50$ there is essontinlly no return flux from the wall botwoon $x=0$ and $x=1.5$. The major portion of the roturn flux from the wall comes from the wall area between $x=1.5$ and $x=10$. For $n=1$ und $\lambda=15$, return flux from the wall close to $\mathrm{x}=0$ is noted, but essentially no wallreturn flux comes from the wall beyond about $x=8$. For $\mathrm{n}=2$, the same $\lambda$-values reprosent more collimated beams; hence, there is less wall-roturn flux from similar facility dimensions.

\section{Concluding Remarks}

A two-parametor equation is shown to be capable of representing experimental ion beam density profilos of bombardment ion thruster exhausts. The equation is a far-field relation (eq. (1)) and thorefore will not be applicable too close to the thruster exit. A calculation of the development of the profiles downstroam of an extended source indicates that ion beam density profiles should be taken at least eight thruster rudil away to bo representative of the far-field bohavior of the ion beam. The equation was shown to represent profiles taken as close as four thruster radil downstream. Ilowover, these may not be representative of the fur-field patterns.

The particular advantage of being able to oxpress the ion beam profile in a concise analytical relation has been illustratod by parametric plots of thrust factor and ion beam envelope curves. Experimental beam profiles illustrated had thrust factors varying from alout 0.95 to nearly 1.0 .

Use of the beam profile equation to calculate backsputtering from vacuum chamber walls during ion thruster testing was illustrated.

\section{Appendix A \\ Symbols}

$c(n, \lambda)$ parameter defined in Eq. (6)

$\mathrm{dA}_{0}$ differential area of thruster, $\mathrm{cm}^{2}$

$\mathrm{dF}_{1,2}$ differential view factor, fraction of emitted flux from surface 1 that intercepts surlace: 2
I(total) total ('urlent, A

J(\%,0) corrent density in molial platue all axial distumee $x$ and radist angle. $1, \lambda / \mathrm{cm}^{2}$

f(\%, r) ourrent density in rabial plater at axial dis-

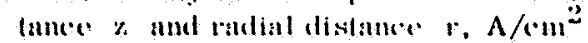

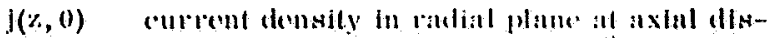

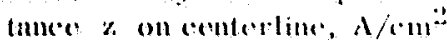

j(0, lo) corrent density in the radial plane at thruster. exit (\%: 0$)$ and at radial distance $r_{0}, \mathrm{~A} / \mathrm{cm}^{2}$

$J(0,0)$ corrent density in radial plane at thruster cxit $\left(x\right.$ o) at conterline, $\mathrm{A} / \mathrm{em}^{2}$

avarago cerrent densily of thruster (edy. (133)), $A / \mathrm{cm}^{2}$

ii(v) mass hlux in molial plane at molius $r$, $\left.\mathrm{kg} /(\mathrm{cm})^{2}\right)(\mathrm{sec})$

n

parameter of lic. (1)

li

radius of latellity valeuum tank (skctch (a)), em

$R_{0} \quad$ ractius of thruster,

r. mallus, $\mathrm{cm}$

ro molius in thruster exit plane, em

s sputtering ylold, atoms/insident lon

sputtoring yiold at normal incidences, atoms/incident ion v

$\mathbf{x}$ velocity

nondincmsional axial distunco, $2 / 12$

nondimensional mallal distancs, $v / x(\approx$ tan $\theta)$

axlal distance variable, c:m

distance of target liom thruater exil plano, em

angle between Hurlace: 10ormal and incident lon direction

angle: in thruster (:xit plane: (sketch (c))

angle from ecenterline of ion hean

angle: at which $\mathrm{j}\left(\%, 0_{0}\right) / \mathrm{j}(\%, 0)$ equal s $0.3(\mathrm{j})$

angle al which $j(\%, 0,0,1) / j(\%, 0)$ equals 0.1

cone half-angle enc:ksting griven fraction of wotal ion heam (oqs. (14) and (15)) 
$\mu_{1}$ particle arrival rate at surface in target plane, atoms $/\left(\mathrm{cm}^{2}\right)$ (sec)

$\mu_{2}$ particle arrival rate at center of thruster exit plane, atoms $/\left(\mathrm{cm}^{2}\right)(\mathrm{sec})$

$\bar{\nu}$ average emitted ion rate from thruster, ions $/\left(\mathrm{cm}^{2}\right)(\mathrm{sec})$

$\nu_{1}$ particle emission rate from target surface, atoms $/\left(\mathrm{cm}^{2}\right)(\mathrm{sec})$

\section{Appendix B}

Development of Far-field Profile from Extended Source

In order to get some indication of the closest approach to an extended source (the thruster) that a farfield expression such as Eq. (1) could be useful, a calculation of the profiles as a function of distance from such a source was carried out.

If it is assumed that nothing influences the ion trajectories once they are beyond the neutralization plane of the thruster, the development of a particular far-field profile, expressible in the form of Eq. (1), would only come about because the local emission sources (for instance, the individual grid holes) exhibit this same type of profile. The amount of emission current from the individual sources may vary. In this particular example a distribution of current density across the radius of the thruster exit $(z=0)$ is assumed to be

$$
\frac{j\left(0, r_{0}\right)}{j(0,0)}=\cos \left(\frac{\pi}{2} \frac{r_{0}}{R_{0}}\right)
$$

That is, individual emitting holes are not assumed, but rather a continuous emission source over the thruster face is assumed. The magnitude of the emission current varies as Eq. (B1) and the distribution locally varies as Eq. (1).

For the profile of Eq. (B1) the current density at the centerline $j(0,0)$ is related to the average current density $\bar{j}$ by

$$
\mathrm{j}(0,0)=2.16 \overline{\mathrm{j}}
$$

where

$$
\overline{\mathrm{j}}=\frac{\mathrm{I}}{\pi \mathrm{R}_{0}^{2}}
$$

A schematic showing the geometric relations is shown in sketch (c).

The flux to point $z, r$ from the differential area of the thruster is

$$
\frac{d j(z, r)}{j\left(0, r_{0}\right)}=c(n, \lambda) \frac{d A_{0}}{\pi z^{2}} e^{-[\lambda(1-\cos \alpha)]^{n}}
$$

By using and

$$
j\left(0, r_{0}\right)=2.16 \bar{j} \cos \left(\frac{\pi}{2} \frac{r_{0}}{R_{0}}\right)
$$

Eq. (B4) can be written

$\frac{j(\mathrm{z}, \mathrm{r})}{\bar{j}}=\frac{2.16 \mathrm{c}(\mathrm{n}, \lambda)}{\pi \mathrm{z}^{2}} \int_{\mathrm{A}_{0}} \cos \left(\frac{\pi}{2} \frac{\mathrm{r}_{0}}{\mathrm{R}_{0}}\right)$
$\exp -\left[\lambda\left(1-\frac{\mathrm{z}}{\sqrt{\mathrm{r}_{0}^{2}+\mathrm{r}^{2}-2 \mathrm{r}_{0} \mathrm{r} \cos \beta+\mathrm{z}^{2}}}\right)\right]^{\mathrm{n}} \mathrm{r}_{0} \mathrm{dr} \mathrm{r}_{0} \mathrm{~d} \beta$

Equation (B7) was integrated numerically. Results for $\mathrm{n}=1$ and $\lambda=20$ are shown plotted in Figs. 8 and 9. Figure 8 shows the variation of $j(z, r) / \bar{j}$ with distance for several $\theta$-values. Values of $j(z, r) / \bar{j}$ approach closely tho se given by the far-field expression within about six to eight thruster radii downstream. The same calculations are presented in a different manner in Fig. 9. This figure shows constant current density lines. The near-field data (dashed lines) blend into the far-field values (solid lines) by about six to eight thruster radii downstream.

\section{References}

1. Ogawa, Howard S.; Cole, Robert K.; and Sellen, J. M., Jr.: Factors in the Electrostatic Equilibrium Between a Plasma Thrust Beam and the Ambient Space Plasma. Paper 70-1142, AIAA, Sept. 1970.

2. Hill, Jacques A. F.; and Draper, James Stark: Analytical Approximation for the Flow From a Nozzle Into a Vacuum. J. Spacecraft Rockets, vol. 3 , no. 10 , Oct. 1966 , pp. 1552-1554.

3. King, H. J.; and Poeschel, R. L.: Low Specific Impulse Ion Engine. Hughes Research Labs. (NASA CR-72677), Feb. 1970 .

4. Reader, Paul D.; Nakanishi, Shiego; Lathem, Walter C.; and Banks, Bruce A.: A Sub-Millipound Mercury Electron-Bombardment Thruster. Paper 70-616, AIAA, June 1970.

5. Nakanishi, S.; Banks, B. A.; and Richley, E. A.: High-Perveance Accelerator Grids for Low-Voltage Kaufman Thrusters. J. Spacecraft Rockets, vol. 5, no. 3, March 1968, pp. 356-358.

6. Byers, David C.: Angular Distribution of Kaufman Ion Thruster Beams. NASA TN D-5844, 1970.

7. Bechtel, Robert T.; Banks, Bruce A.; and Reynolds, Thaine W.: Effect of Facility Backsputtered Material on Performance of Glass-Coated Accelerator Grids for Kaufman Thrusters. TM X-52927. Presented at AIAA 9th Aerospace Sciences Meeting, New York, N.Y., Jan. 25-27, 1971. 
8. Reynolds, Thaine W.; and Richley, Edward A.: Free-Molecule Flow and Surface Diffusion Through Slots and Tubes - A Summary. NASA TR R-255, 1967.
9. Wehner, Gottfried: Influence of the Angle of Incidence on Sputtering Yields. J. Appl. Phys., vol. 30, no. 11, Nov. 1959 , pp. 1762-1765. 
$m$
0
0
1
1
I

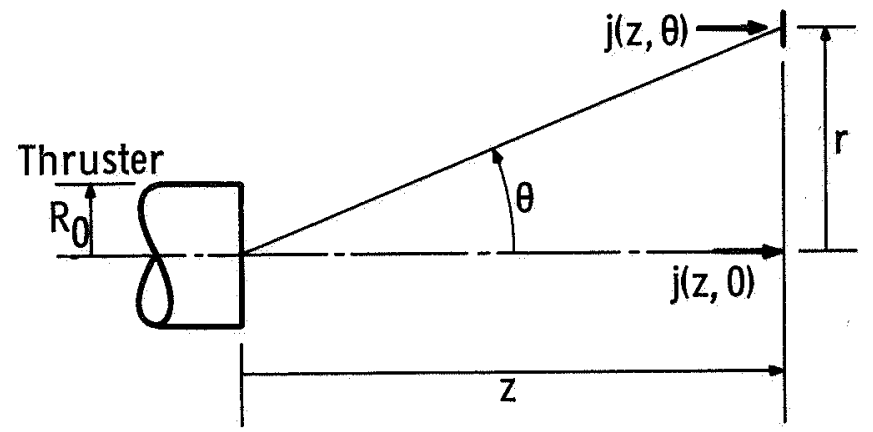

(a)

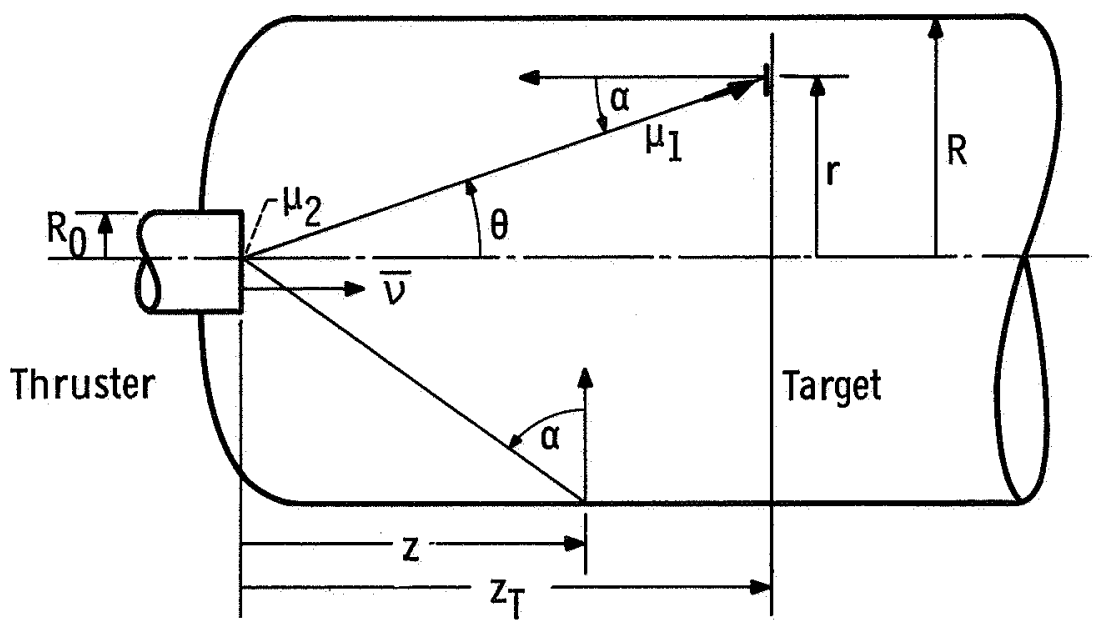

(b) 


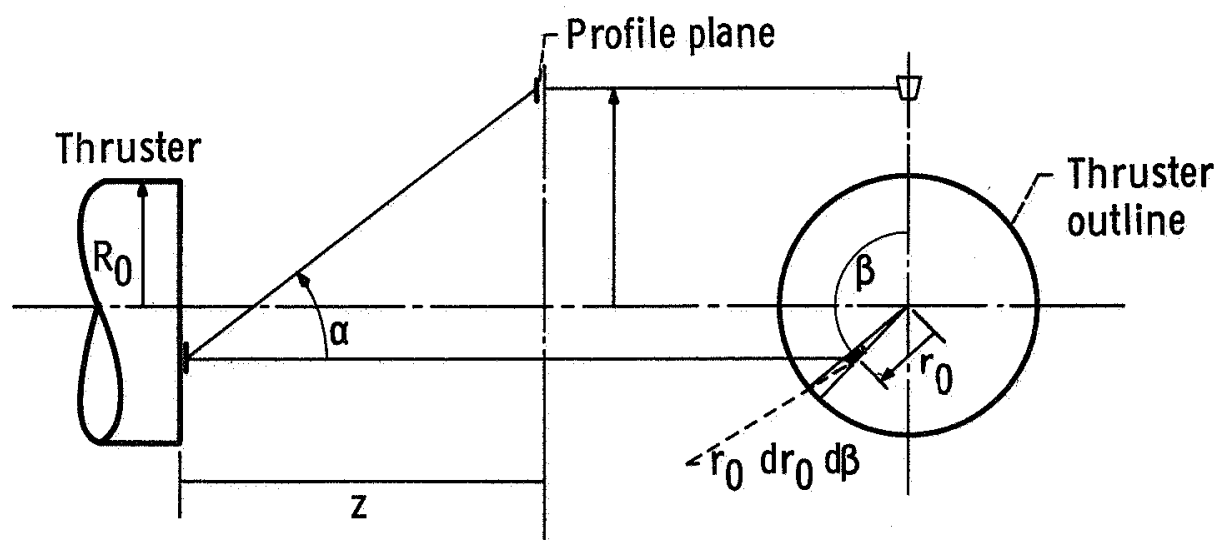

(c)

TABLE I. - COMPARISON OF ION BEAM PROFILES WITH EQUATION (1)

\begin{tabular}{|c|c|c|c|c|c|c|c|}
\hline \multicolumn{4}{|c|}{ Thruster identification } & \multirow{2}{*}{$\begin{array}{c}\text { Axial distance } \\
\text { of profile, } \\
z / R_{0}\end{array}$} & \multicolumn{2}{|c|}{ Constants } & \multirow{2}{*}{$\begin{array}{l}\text { Thrust } \\
\text { factor, } \\
\text { F }_{\mathbf{T}}\end{array}$} \\
\hline $\begin{array}{l}\text { Diameter, } \\
\text { cm }\end{array}$ & Type & Figure & $\begin{array}{l}\text { Refer - } \\
\text { ence }\end{array}$ & & $\mathbf{n}$ & $\lambda$ & \\
\hline 30 & Glass grid & $3(a)$ & 3 & 10 & 1 & 24.3 & 0.954 \\
\hline 5 & Glass grid & $3(b)$ & 4 & 23.3 & .02 & 40.2 & .966 \\
\hline 5 & Glass grid & $3(c)$ & (a) & 4 & 1. 28 & 18.2 & .951 \\
\hline \multirow[t]{2}{*}{5} & Two grid & $3(d)$ & 5 & 14.8 & .9 & 63.3 & .980 \\
\hline & & & & 25.2 & .9 & 63.3 & .980 \\
\hline (b) & (b) & 3(e) & 1 & $\cdots$ & 1. 19 & 85 & .99 \\
\hline \multirow[t]{2}{*}{15} & Two-grid & $3(f)$ & 6 & 4 & 1.23 & 34.6 & c.976 \\
\hline & & & & & 1.4 & 17.0 & d. 955 \\
\hline 30 & Glass grid & $3(\mathrm{~g})$ & 7 & 4.8 & .8 & 42.9 & .960 \\
\hline
\end{tabular}

${ }^{a}$ Unpublished data by J. H. Molitor and H. J. King of Hughes Research Laboratory, Malibu, Calif. $\mathrm{b}_{\text {Not given in reference. }}$

${ }^{c}$ Case III of ref. 6 .

${ }^{\mathrm{d}}$ Case I of ref. 6 . 
$m$
0
1
1
1
a.
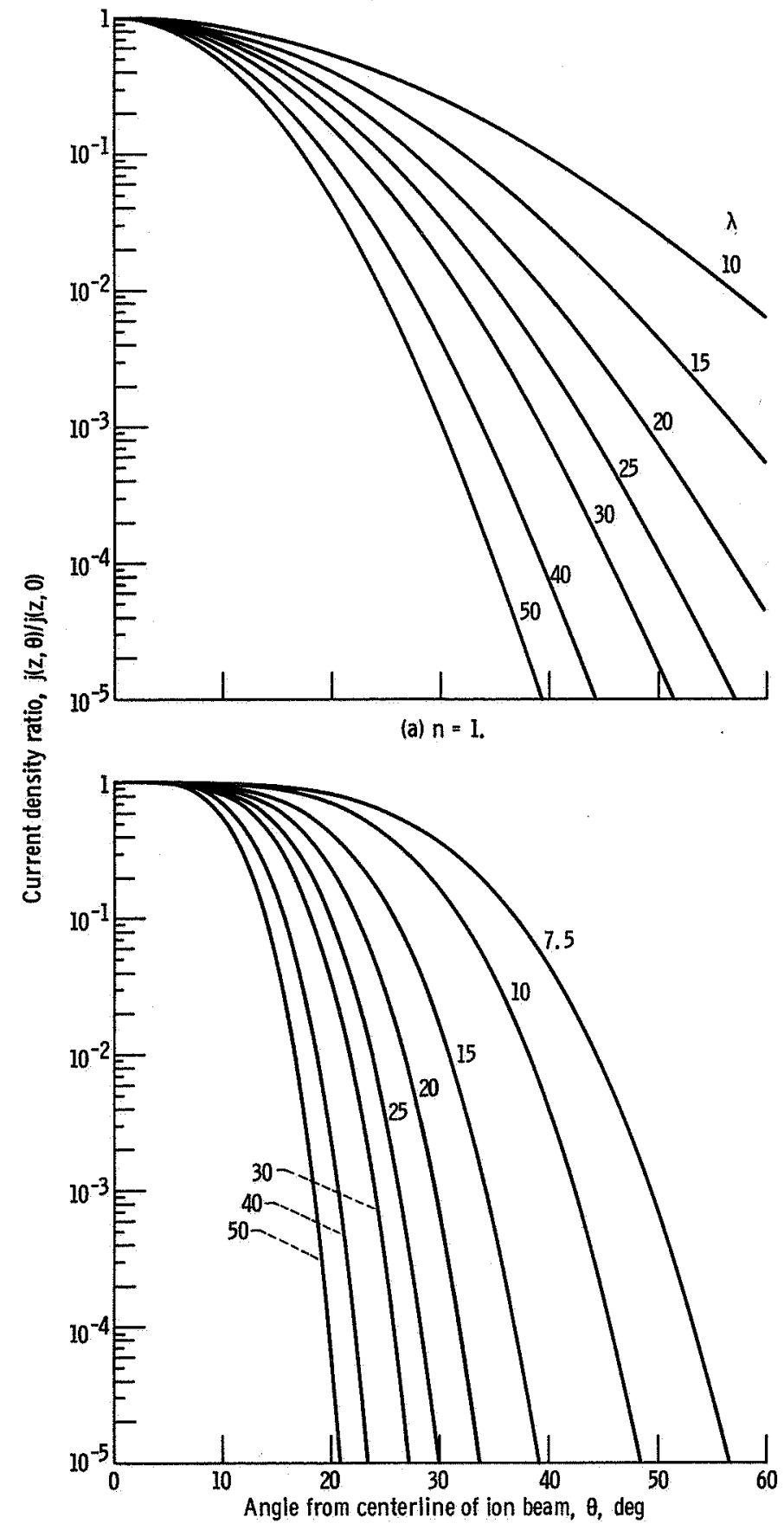

(b) $n=2$.

Figure 1. - Beam density profiles. (Eq. (1).) 
$m$
0
0
1
1
c1

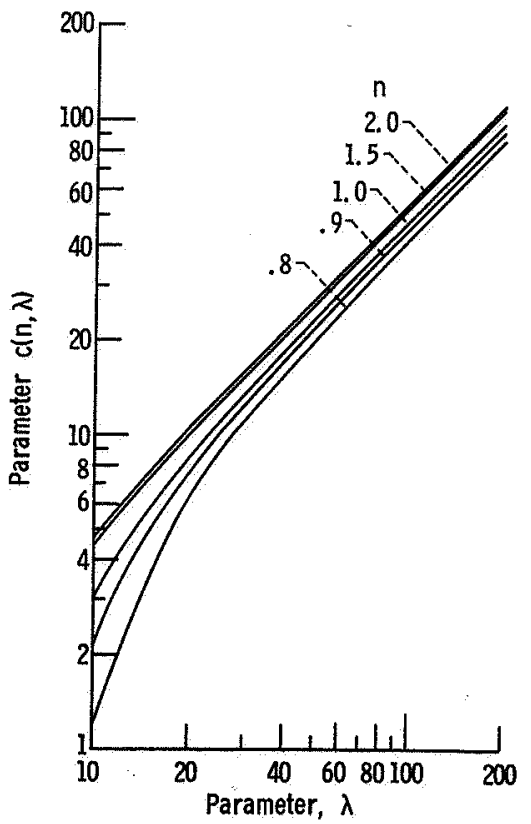

Figure 2. - Value of parameter $c(n, \lambda)$. (Eq. (6).) 

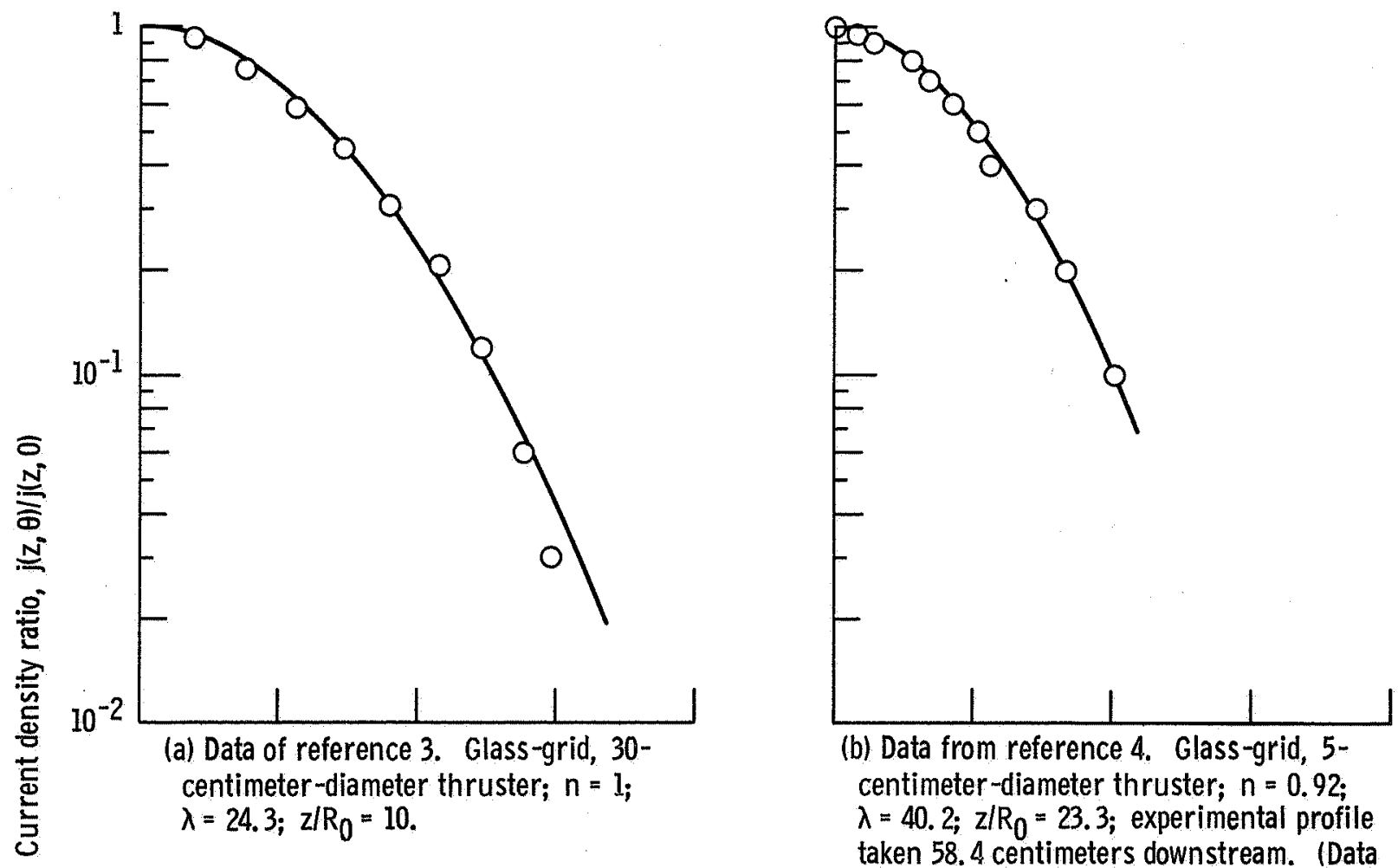

(b) Data from reference 4. Glass-grid, 5centimeter-diameter thruster; $n=0.92$; $\lambda=40.2 ; z / R_{0}=23.3$; experimental profile taken 58.4 centimeters downstream. (Data from fig. 10 of ref. 4).
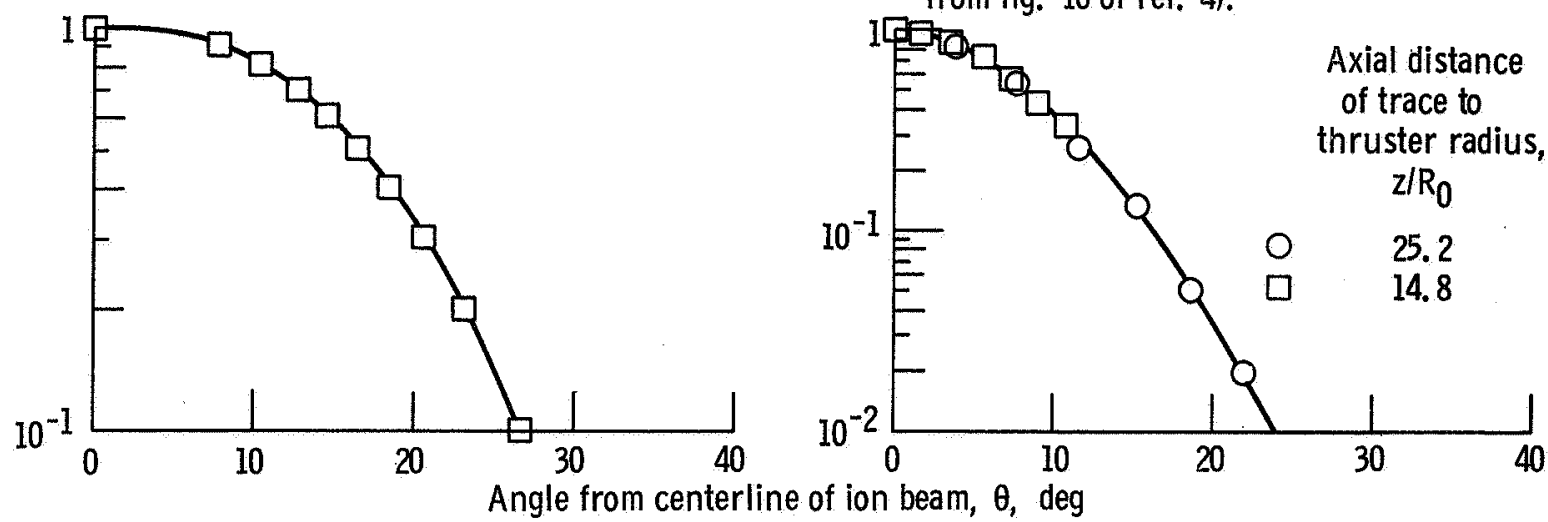

(c) Unpublished data by J. H. Molitor and H. J. King of Hughes Research Laboratory, Malibu, Calif. Glass-grid, 5-centimeterdiameter thruster; $n=1.28 ; \lambda=18.2$.

(d) Data from reference 5. Glass-grid, 5centimeter-diameter thruster; $n=0.9$; $\lambda=63.3$.

Figure 3. - Comparison of profile equation (1) with data. Solid line is equation for $n, \lambda$ values cited. 

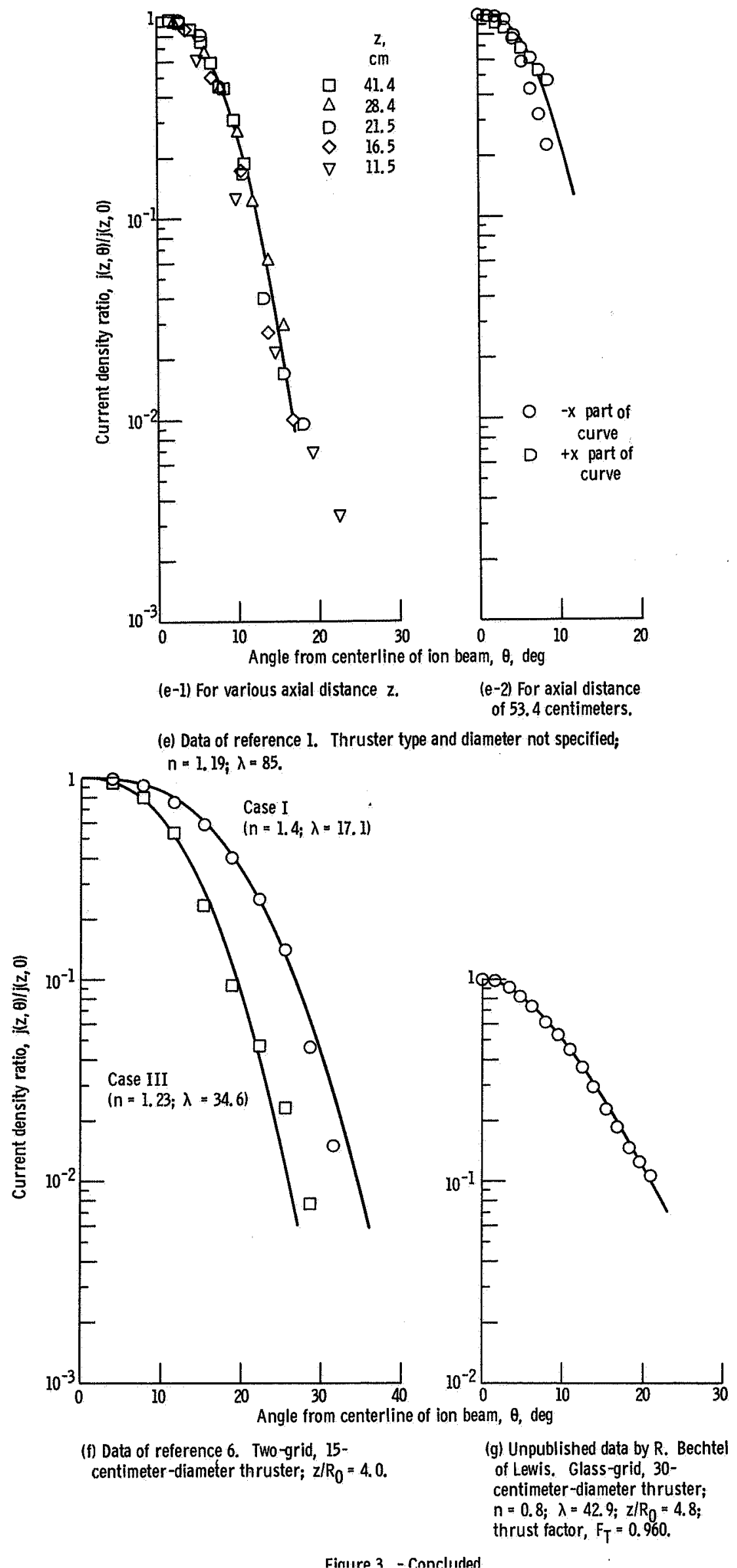

Finure 3 - Concluded 

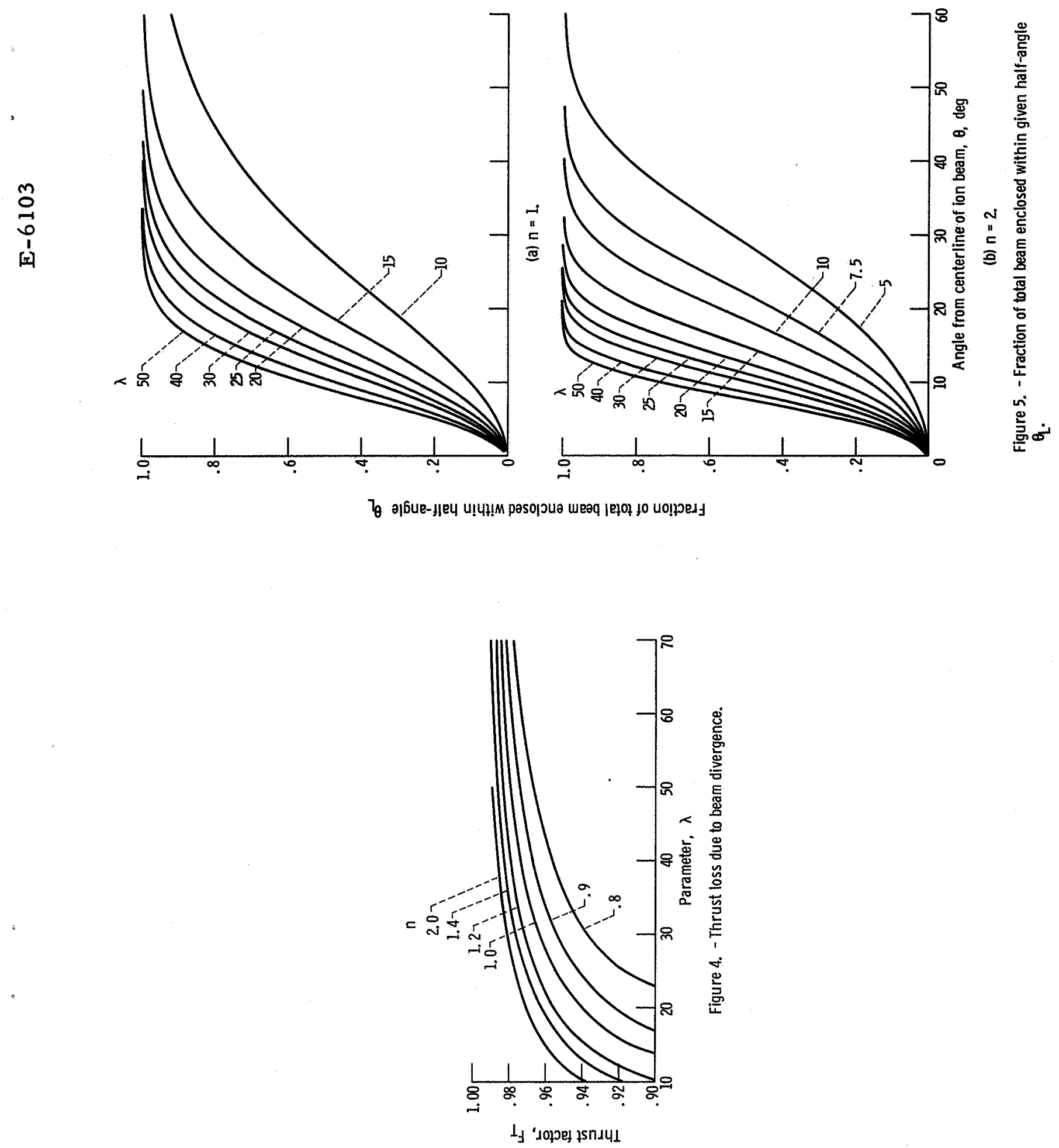
$m$
0
0
1

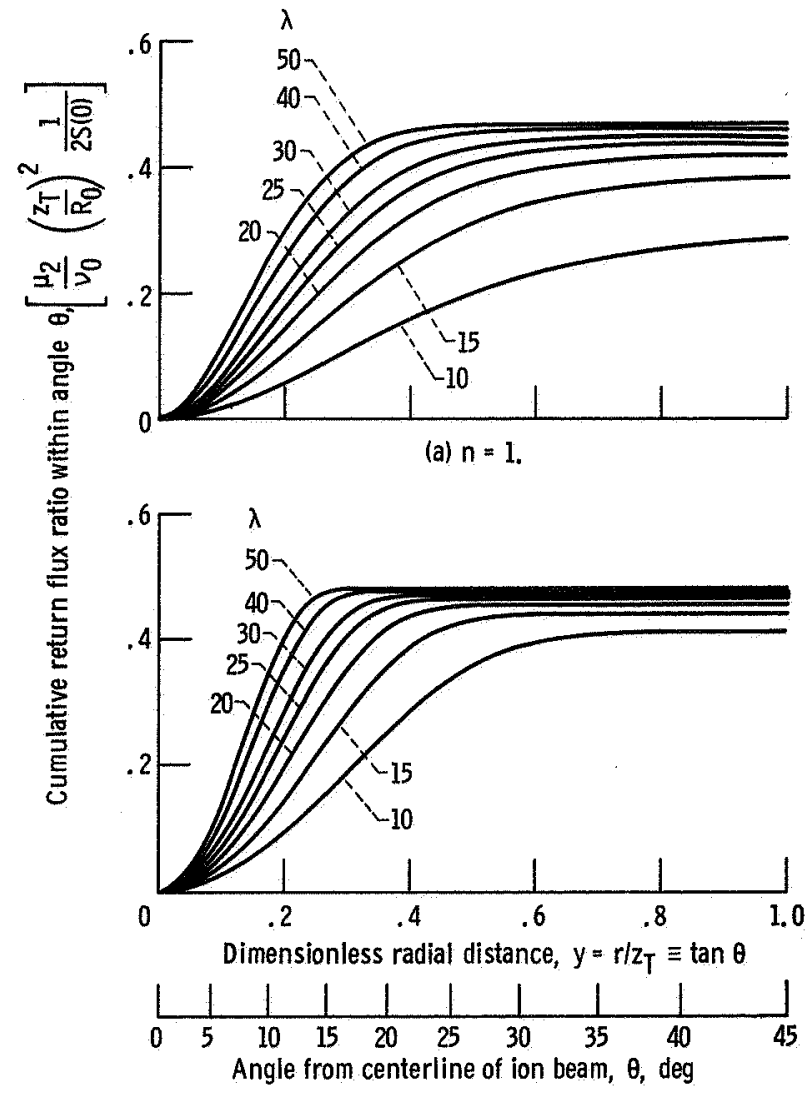

(b) $n=2$.

Figure 6. - Return flux from circular target. 
$m$
0
0
0
1
1

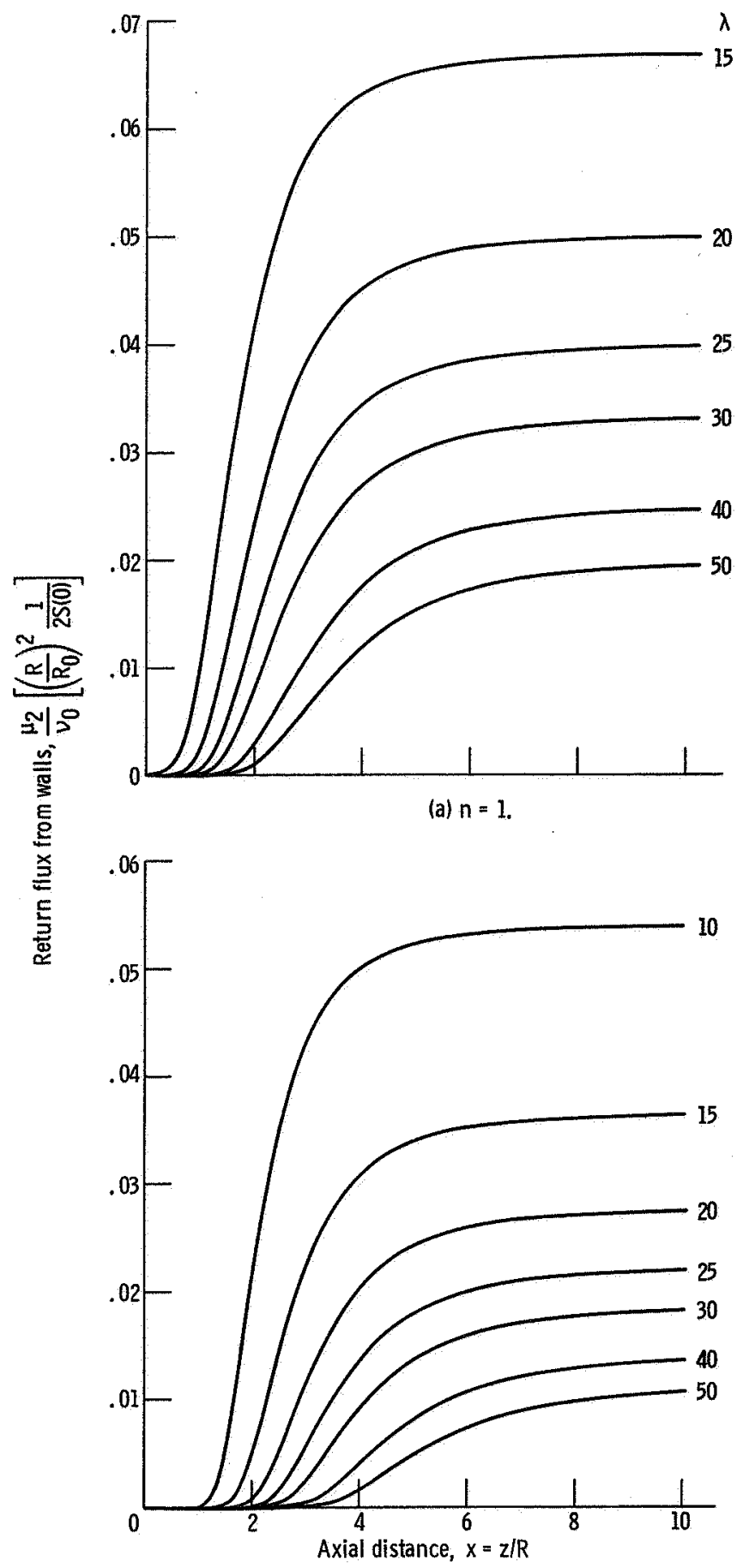

(b) $n=2$.

Figure 7. - Return flux from tank wall. 


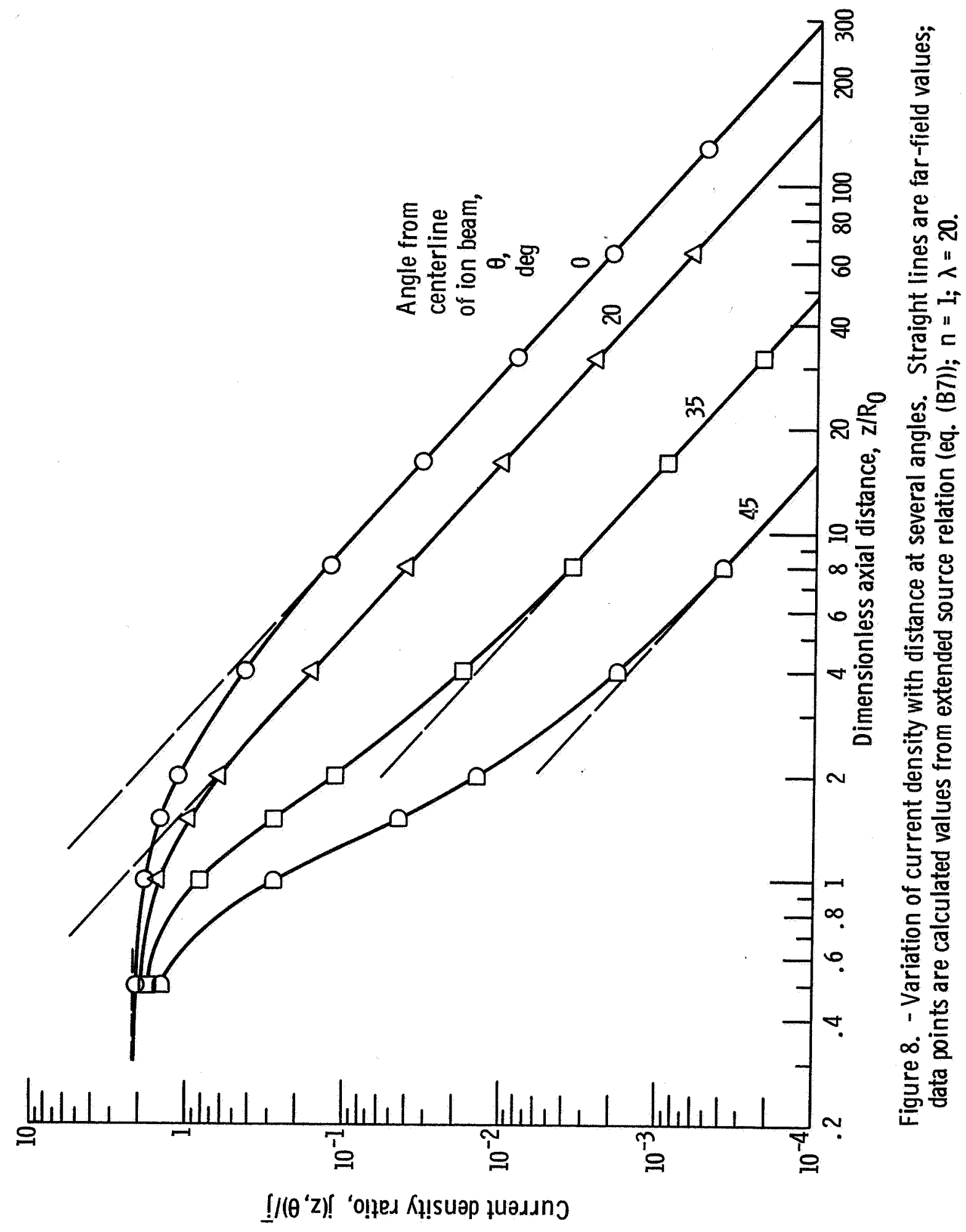


$n$
0
0
1
1
01

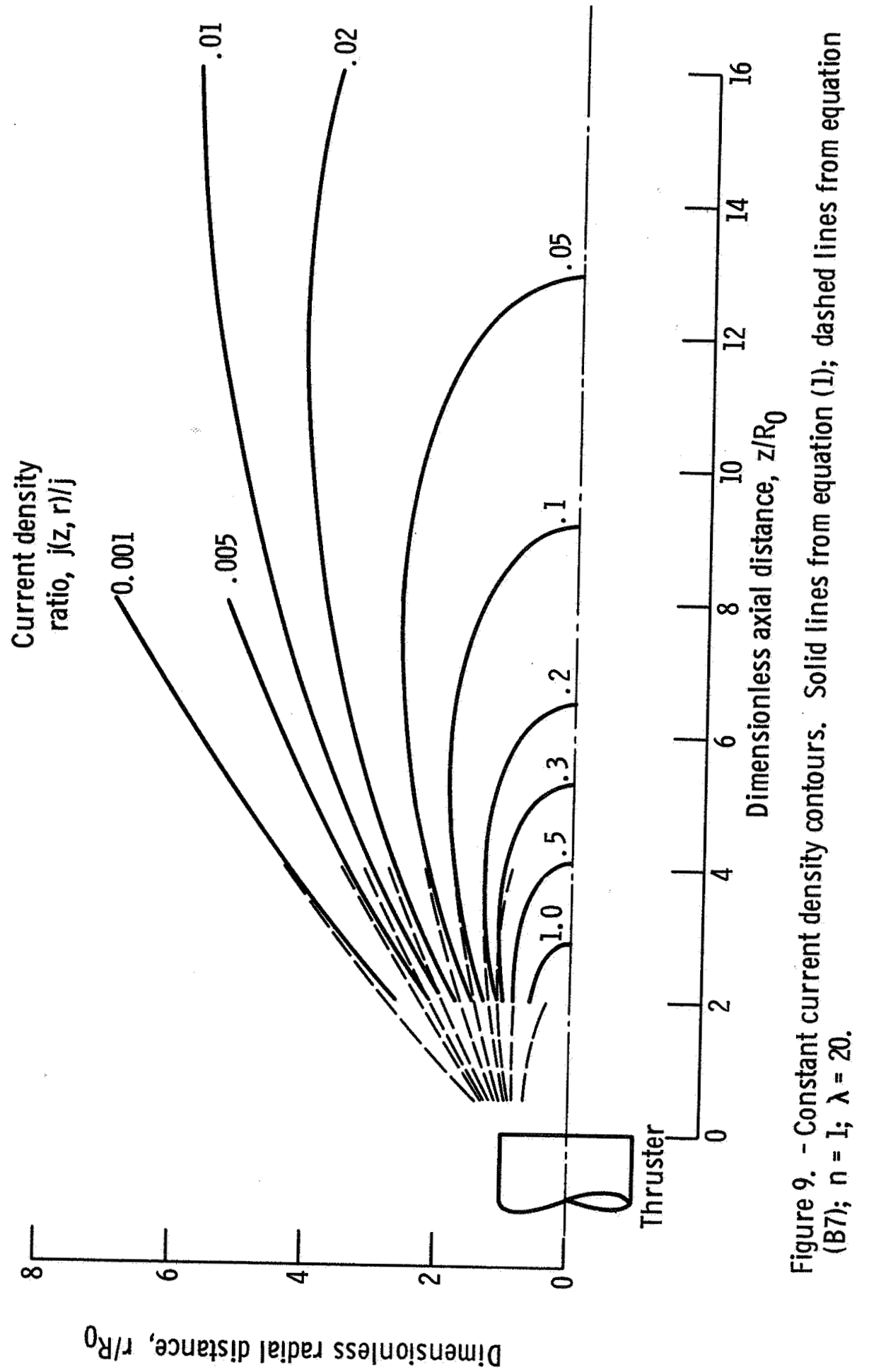

NASA-Lowis-Com'I 